Aims. To survey the prevalence of monitoring of vitamin D on an inpatient ward.

To audit the treatment if there is identified vitamin D deficiency or insufficiency

To compare differences between findings in audits

Method. All inpatients admitted to Milford centre between August 2019 and August 2020 were selected as part of the sample size.

Data were collected by FY1 and FY2

Patients' laboratory results were accessed to determine vitamin D levels.

E-notes were used to conclude who were vitamin D sufficient or deficient for treatment

The standard for the audit were as per:

Management of vitamin D deficiency or insufficiency in adults CKS (2018)

The above was based on National Osteoporosis Society (NOS) guideline Vitamin D and bone health: a practical clinical guideline for patient management [National Osteoporosis Society, 2013] and Scientific Advisory Committee on Nutrition (SACN) guideline Result. 2017

$48 / 188$ patients had vitamin D levels measured

$36 / 48$ patients had sufficient vitamin D levels

$12 / 48$ patients were either deficient or insufficient

$12 / 12$ patients were treated where found deficient or insufficient

2020

90/115 patients had vitamin D levels measured

$47 / 90$ patients had sufficient vitamin D Levels levels

$43 / 90$ patients had either insufficient or deficient vitamin D

22/43 patients had treatment documented in noted where found deficient or insufficient

Conclusion. Difficult to make comparisons with previous audit due to difference in number of patients tested

Vitamin D is routinely tested on Milford ward on admission hence the large number compared to the last audit

$52 \%$ had noted to have sufficient levels of vitamin D

Concerning were results that only $51 \%$ of those deemed to have insufficient or deficient were treated based on notes

Potential reasons could be:

Prescribed in medication card and not documented in notes.

Vitamin D results checked in another ward, no supplementation given, and then transferred to Milford house.

Patients refused treatment but not documented adequately.

Patient discharged before results were received due to quick around

Results were deemed insufficient in terms of the range but very close to normal hence decision made not to start supplementation

Results to be disseminated with medical and nursing colleagues

Re-audit in September 2021

\section{Off-label prescribing of quetiapine in south locality} crisis teams

Mamta Kumari ${ }^{1 \star}$, Arun Kumar Gupta ${ }^{2}$ and Peter Clarke ${ }^{3}$

${ }^{1}$ Roseberry Park Hospital; ${ }^{2}$ Sunderland South CTT and ${ }^{3}$ South

Pharmacy Department, Hopewood Park

${ }^{\star}$ Corresponding author.

doi: 10.1192/bjo.2021.267

Aims. The audit was carried out to determine the frequency of off label prescribing of quetiapine and compliance with standards within Trust Policy (UHM PGN 02 PPT PGN 08) - Physical
Health Monitoring of Patients Prescribed Antipsychotics and other Psychotropic Medicines, NICE CG178, General Medical Council Ethical Standards and Royal College of Psychiatrists College Report CR210.

The main objectives of the audit were to determine if:

Patients have been appropriately informed of off-label status and consent recorded.

Alternative licensed treatment first used/ruled out.

Appropriate communication on transfer of care.

Appropriate physical health monitoring completed.

Background. Quetiapine is associated with various physical side effects. Patients should be fully informed of the expected risks and benefits of treatment, and the limited evidence base for offlabel prescribing.

There are additional issues around the transfer of prescribing to primary care.

Method. The sample consisted of 50 consecutive patients selected from the crisis team caseload in the month of December 2018.

Data reviewed in this audit were taken from six months period.

Records audited were obtained from $\mathrm{RiO}$ (electronic records) and prescription charts.

Data collection was started in January 2019 and completed in March 2019

The audit tool was a dichotomous scale questionnaire based on NICE guidelines.

Result. 4 patients from the sample (8\%) were prescribed off-label quetiapine.

$100 \%$ had physical health monitoring completed as per Trust policy.

$100 \%$ off-label indication been clearly documented in notes.

$100 \%$ Consent to treatment was documented.

$100 \%$ had medication reviewed in the previous 6 months.

$75 \%$ had licensed medication used or ruled out before considering off-label quetiapine use

$25 \%$ risks/benefits of treatment were documented as part of a patient discussion.

25\% had documented evidence that alternative treatment options were discussed.

25\% had documented evidence of Community consultant/GP consent/agreement was obtained before transfer of prescribing

$75 \%$ had a documented plan for review of quetiapine for treatment efficacy and side effects

$50 \%$ had a documented plan in place for ongoing physical health monitoring

Conclusion. Suggested a wider audit may be required with greater patient numbers and which specifically filters for patients prescribed quetiapine.

Audit result has been shared with Crisis team members, Medicines Optimisation Committee and South Locality Quality Standards Committee in the trust.

Clinical audit of cardio-metabolic monitoring in people with intellectual disability (PWID) taking antipsychotic medication

Sandar Kyaw*, Fadzlien Bintizahari and Peter Speight

Lincolnshire Partnership Foundation Trust

${ }^{\star}$ Corresponding author.

doi: $10.1192 /$ bjo.2021.268

Aims. To ensure close monitoring of physical health parameters when antipsychotics are prescribed and to liaise with primary care to ensure appropriate interventions are implemented. 
Background. Antipsychotics are the most frequently prescribed psychotropic medication for PwID. Treatment with antipsychotic agent is associated with cardio-metabolic risks such as obesity, diabetes, and dyslipidemia. A strong association is well documented between antipsychotic use and the risk of stroke in schizophrenia although the magnitude of this association has yet to be studied in PwID.

PwID have an increased risk of premature death. Cardio-metabolic monitoring and appropriate intervention to this vulnerable cohort will improve the preventable cardio-metabolic multimorbidity. The NICE guideline (CG11) recommends antipsychotic medication should only be initially prescribed and monitored by the secondary care professionals for at least 12 months. They also should work together with primary care to ensure appropriate interventions are arranged where necessary.

Method. A retrospective audit was performed for 40 service users, taking antipsychotic medication. Quota sampling was used to identify 10 cases each from the caseload of 4 consultant psychiatrists, within the Intellectual Disability community setting, between September 2019 and October 2019.

An audit tool was designed, in accordance with cardiometabolic measures (smoking status, height, weight, Blood Pressure, HbA1c, Lipid profile), based on physical health CQUIN targets and the Lester adaptation tool. Collection of data was performed from electronic case records and electronic blood results service. The work was performed with the approval of local clinical audit team and analysed by using Microsoft Excel. Result. Baseline cardio-metabolic assessment was observed in over a half of the sample population (50-65\%) whilst only less than $15 \%$ was noted at 3-6 months. Documentation Evaluation of physical health assessments for new admissions to the Oleaster during the first wave of COVID-19 on body weight and blood pressure was seen only in $15 \%$ and $2.5 \%$ of population respectively at 3-6 months. Collaboration with GP for annual health check was observed in $78-100 \%$ of population.

Intriguingly, our finding indicates a significant improvement in all required compliance when nursing team is involved.

Conclusion. Improving physical healthcare is essential to reduce the cardio metabolic outcome in PwID taking antipsychotic medication. Better involvement of community nurses as well as availability of Sphygmomanometers at every outpatient clinic will determine the successful implementation of cardio metabolic monitoring and effective collaboration with primary care clinicians.

Once the action plan is disseminated to the teams, the impact of change will be reassessed by a re-audit in one year's time.

\section{Evaluation of physical health assessments for new admissions to the Oleaster during the first wave of COVID-19}

Erin Lawson-Smith ${ }^{1 \star}$, Danielle Sutherland ${ }^{1}$, Eleanor Brookes ${ }^{2}$, Alex Zhang ${ }^{2}$ and Joji George ${ }^{2}$

${ }^{1}$ University of Birmingham, Medical School and ${ }^{2}$ Birmingham and Solihull Mental Health Foundation Trust

${ }^{*}$ Corresponding author.

doi: 10.1192/bjo.2021.269

Aims. Physical health of psychiatric inpatients is worse than the general population. Physical health monitoring of these patients can have positive effects on outcomes. Birmingham and Solihull Mental Health Foundation Trust (BSMHFT) states that a physical health assessment (PHA) should be completed within 72 hours of admission. This comprises a physical health form (PHF) and minimum data set (MDS): BP, BMI, TB and BBV status, alcohol and drug screen, smoking status, Hba1c and lipids. In a 2017 audit, compliance was shown to need improvement, with $28.3 \%$ of admissions not having a PHF documented.

Objectives. To assess whether PHAs for new admissions to the Oleaster, Birmingham during the first wave of COVID-19 were completed in line with trust policy

To compare findings with a previous audit

To make recommendations to improve inpatient physical health and compliance with trust policy

Method. A retrospective audit was conducted, with PHA details accessed via the electronic medical records system $\mathrm{RiO}$. Admissions from 16/03/2020-30/06/2020 were accessed and 158 admissions (155 patients) were included. 21 admissions were excluded as they were internal transfers; only data from the initial admission were included. Data were collected by 2 medical students and a psychiatry trainee using a data collection tool. Data were recorded and analysed on Excel.

Result. Of 158 admissions, 81 had PHFs (51.3\%). 59 were completed within 72 hours of admission (34.3\%); 39 were completed fully $(24.7 \%)$. Of incomplete PHFs, 2 explicitly stated incompletion due to COVID-19. 22 PHFs were created but not completed within 72 hours. 15 gave a deferral reason e.g., refusal to consent or agitation. For 77 admissions (47.3\%), no assessment was documented, with no reason given.

2 admissions (1.3\%) recorded the full MDS within 72 hours of admission.

2 admissions (1.3\%) had fully complete PHAs (PHF and MDS) within 72 hours of admission, fulfilling trust policy.

Conclusion. $51.3 \%$ of admissions had a PHF, with $34.3 \%$ documented within 72 hours of admission. However, only $1.3 \%$ of admissions fulfilled trust policy of both a completed PHF and MDS within 72 hours of admission. There were more admissions without a PHF than in the previous 2017 audit; $47.33 \%$ compared to $28.3 \%$ previously. Given trust targets that a PHA should be fully completed for $100 \%$ of admissions, it was found that the Oleaster did not meet these guidelines during this period and improvements must be made to maintain integrity of patient care.

\section{Impact of COVID-19 on referrals for physical and} mental health care

Petros Lekkos ${ }^{1 \star}$, Joanna Thorpe ${ }^{2}$, Janet Obeney-Williams ${ }^{1}$ and Fiona Xing ${ }^{2}$

${ }^{1}$ Camden and Islington NHS Foundation Trust and ${ }^{2}$ Whittington Health NHS Trust, Camden and Islington Foundation NHS Trust ${ }^{*}$ Corresponding author.

doi: 10.1192/bjo.2021.270

Aims. To determine the effect of the COVID-19 pandemic on referrals to mental health and physical health services.

Method. We analysed referral data from three psychiatric services in the boroughs of Camden and Islington across 2018-2020: Early Intervention Services (for patients with a 1st episode of psychosis), Crisis Resolution Teams and inpatient admissions. We also analysed GP referral data to Cancer Services (two-week wait referrals) to Whittington Hospital, Royal Free Hospital and University College Hospital (all of North Central London). We examined the impact of the COVID-19 pandemic on these referrals and compared the findings between physical and mental health. We chose to use EIS and Cancer services as comparable services since they both operate with the two-week target of achieving diagnosis of psychosis and cancer respectively. 